\title{
The 27-Day Recurrence Tendency in Cosmic Ray Intensity and Its Correlation with Solar and Geomagnetic Activity
}

The existence of a 27-day recurrence tendency in cosmic ray intensity had been pointed aut by many authors with the aid of Chree's superposed epoch method. Simpson et al (1), observing the neutron intensity at Climax in 1951, found the existence of a 27-day periodicity in the intensity from the direct measurements.

Recently, a typical 27-day variation of the intensity has been found which continued through 18 solar rotations, from August 27, 1962 to December 25, 1963. This variation will be reported in what follows, in connection with the solar and terrestrial phenomena.

During the above-mentioned period, a clear 27-day recurrence tendency has been observed in the geomagnetic $K p$-index. The 27-day diagram of $\Sigma K p$ (daily sum of $K p$ ) in Fig. 1 shows large activities recurring in the epoch of about the fifth to the eleventh day in each rotation cycle. The activity of the geomagnetic pulsation $(p c 2,3)$ at Onagawa(2) also shows a strong 27-day recurrence tendency in the epoch. Corresponding to such a time pattern of magnetic activity, daily mean value of neutron intensity $I_{n}$ at Deep River has decreased during the same epoch in each cycle, as shown in Fig. 2.

The averaged 27-day variations of $I_{n}$ and $\Sigma K p$ over the whole period in Fig. 3 show their mutual relation more clearly during the epoch. Daily values of solar radio flux density at various frequencies from 200 to $9400 \mathrm{Mc} / \mathrm{s}$ do not show any anomalous change near the epoch and only one of them is shown in the figure. The coronal green line emission indices ${ }^{(3)}$ $G_{6}$ for northern hemisphere and the relative sunspot number $R$ show a hump during the epoch and seem to be related to the recurrence tendency. 1t must be emphasized further that the indices show a similar trend in almost every rotation cycle, whereas $R$ does not.

The long sequence of magnetic activity in Fig. 1 suggests the existence of so-called $M$-region ${ }^{(4)}$ on solar surface. Solar stream is supposed to be activated by this region and to produce the decrease of cosmic ray intensity. Recently, much information about the solar stream has been accumulated by satellite's measurements. Analyzing the Mariner II obtained data during the period August 29, 1962 through January 3, 1963, Snyder et al(5) pointed out that the interplanetary plasma velocity $V$ showed a very strong 27 -day recurrence tendency and had a close correlation with $\Sigma K p$. They also showed 6 sequences of recurrence for $V$ and $\Sigma K p$, both being well correlated to each other, and discussed them in relation to $M$-region. The period during which their data were available is just the early stage of the period for the present analysis and the longest sequence $A$ in their paper is the very initial part of the present one.

As mentioned above, the averaged 27-day variations of $\Sigma K p$ and $I_{n}$ or the meson intensity $I_{m}$ have a good correlation to each other during the epoch. On the contrary, Snyder et al found, in their analysis, rather poor correlation between them. This is really true also 


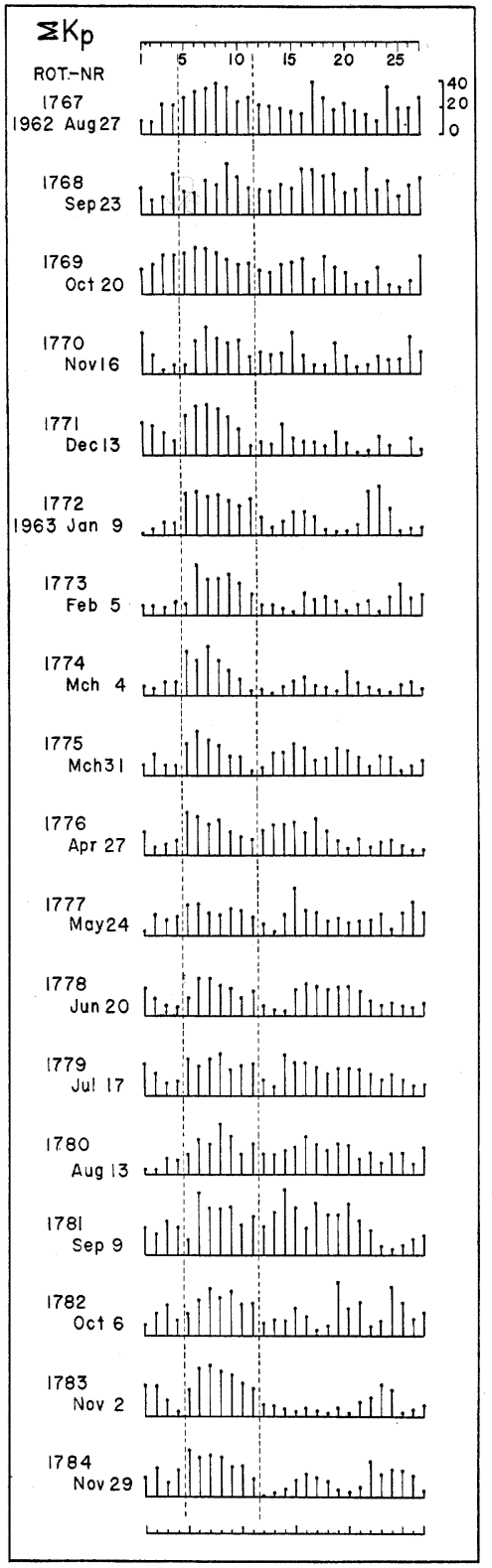

Fig. 1. 27-day diagram of the geomagnetic $\sum K p$-index from August 27, 1962 to December 25, 1963.

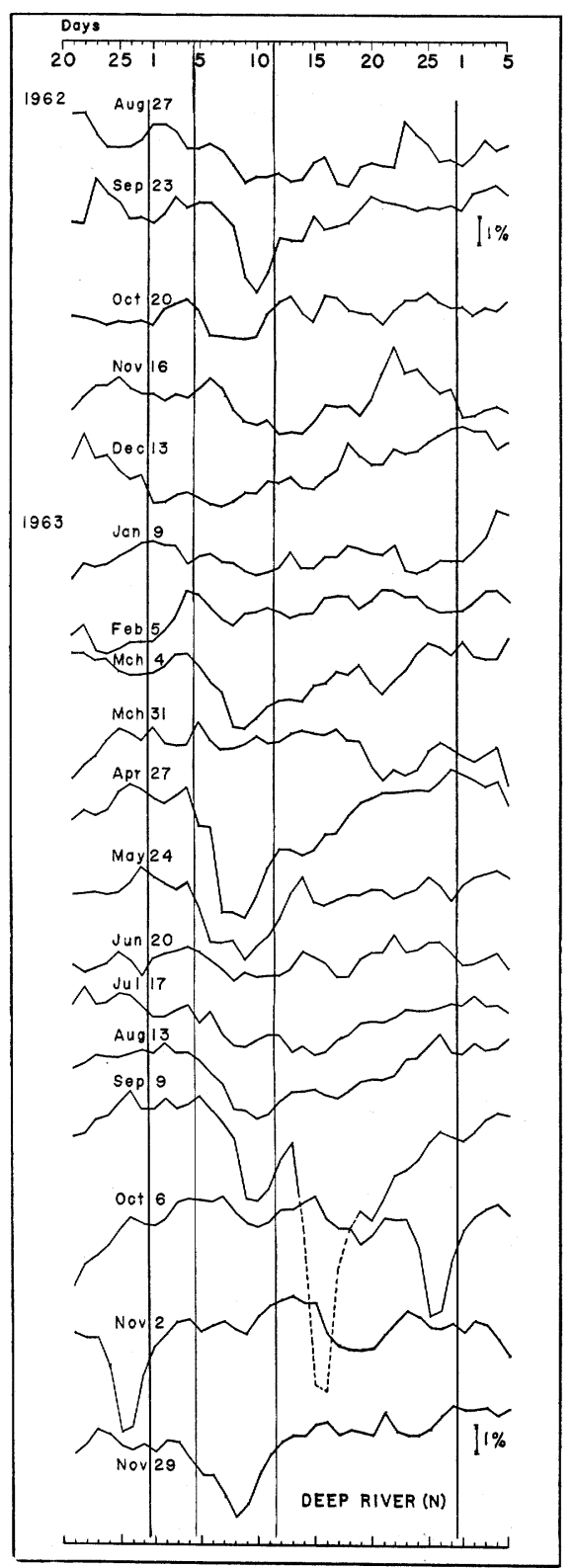

Fig. 2. 27-day variation of daily mean value of neutron intensity at Deep River from August 27, 1962 to December 25, 1963. 
Fig. 3. The averaged 27-day variation of $\sum K p$, cosmic ray intensity $I$, coronal green line index $G_{6}$, relative sunspot number $R$, and solar radio flux density (3750 Mc/s) from August 27, 1962 to December 25, 1963.

$I_{n}$ : neutron intensity at Deep River corrected for barometer effect,

$I_{m}$ : cubic meson intensity at Tokyo corrected for barometer effect and further for decay effect (The last 18th rotation's is not included.),

$\left(G_{6}\right)_{N}$ and $\left(G_{6}\right)_{S}$ : mean of six highest line intensities on northern and southern hemisphere at central meridian,

$N E$ and $S E$ : values on the limb in north-east and south-east quadrants observed 7 days earlier,

$N W$ and $S W$ : values on the limb in north-west and south-west quadrants observed 7 days later.

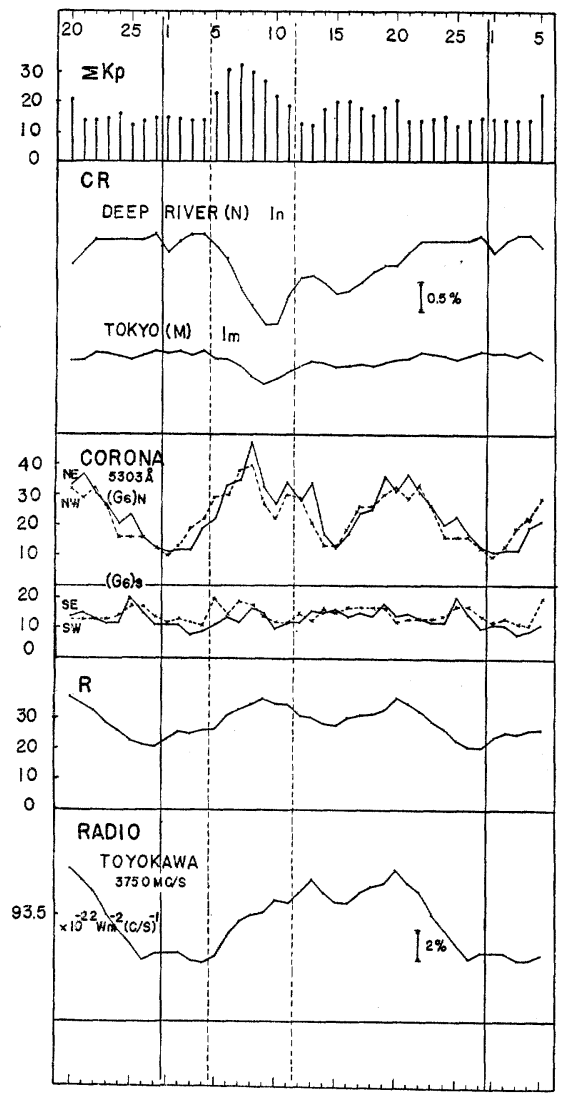

for the present case if their mutual relation is examined more quantitatively by taking account of their values of each epoch. However, under the present condition that the relation between cosmic ray intensity $I$ and $M$-region is not yet well-known, it is worthwhile to point out that the former has decreased positively during the epoch in every rotation cycle and its overall trend is consistent with that of $\Sigma K p$.

Since the coronal green line emission indicesh ad good correlation with $\Sigma K p$ and $I$ in the epoch, a region where the line was kept intensified might be identified with $M$ region. By the present analysis, its position can be limited in the northern hemisphere and its limits in latitude could be narrowed further by careful examination of the original data of green line instead of indices.

Greenstadt ${ }^{(6)}$ found a close correlation of coronal green line daily mean index to the interplanetary magnetic field near the earth by considering the seven day delay of the field from the index. As shown in Fig. 3, the time delay of $I$ and $\Sigma K p$ from $\left(G_{6}\right)_{N}$ is at most one day. Such a short time delay may be due to a stream velocity higher than Greenstadt's. The estimated value of $V$ from the formula given by Snyder et al is about $590 \mathrm{~km} / \mathrm{s}$, corresponding to the maximum of $\Sigma K p$. It takes about three days with this velocity for the stream to reach the earth's orbit from the sun. This seems to be contradictory to the observed time delay of about one day. But, such an inconsistency can be removed easily by assuming that the solar stream does not flow out only radially from the source but also 
within a cone of a considerably large solid angle. Let the half-angle of the cone and the stream velocity be respectively 3 (day) $\times 2 \pi / 27$ (rad./day) and $\sim 590 \mathrm{~km} / \mathrm{s}$, the disturbance by the stream is expected near the earth at the same time as the central meridian passage $(C M P)$ of the source. As the maximum disturbance is likely to occur near the region just behind the front rather than the geometrically central part of the stream, its time delay from $C M P$ of the source could be less than 3 days. Detail analysis will be published later. Finally, the authors wish to thank Dr. H. Carmichael (Chalk River), Dr. Y. Miyazaki (Tokyo) and Prof. A. Kimpara (Toyokawa) for providing the cosmic ray and solar radio data.

\section{References}

(1) Simpson, J.A., Fonger, W. and Wilcox, L., Phys. Rev., 85, 336 (1952).

(2) Saito, T., Tohoku University, Sendai, Japan (private communication).

(3) CRPL-F, Part B, Aug. 1962 to Feb. 1964, National Bureau of Standards, Central Radio Propagation Laboratory, Boulder, Colorado.

(4) Bartels, J., Terr. Mag. and Atm. Elec., 37, 48 (1932).

(5) Snyder, C.W., Neugebauer, M. and Rao, U.R., J. Geophys. Res., 68, 6351 (1963).

(6) Greenstadt, E.W., Astrophys. J., 137, 999 (1963).

By Satoru MorI Hirosachi Ueno Kazuo Nagashima

Department of Physics, Nagoya University

and Syuji SAgisakA

Department of Physics, Shinshu University 